\title{
Models for the evolution of selfing under alternative modes of inheritance
}

\author{
ROBERT LATTA* \& KERMIT RITLAND \\ Department of Botany, University of Toronto, Toronto, Ontario, Canada, M5S $3 B 2$
}

\begin{abstract}
In an approach that explicitly incorporates genetic changes over consecutive generations of selffertilization, we contrast the effect of different modes of inheritance for selfing rate upon the evolutionary equilibrium selfing rate. A monotonic decline of fitness with consecutive generations of selfing was assumed. We found the range of conditions permitting evolutionarily stable mixedmating systems to increase with the number of genes controlling the selfing rate. When the mating system is controlled by a single locus with alternative alleles for complete selfing or outcrossing, alleles for selfing concentrate in inbred individuals and are exposed to stronger selection than the average inbreeding depression. By contrast, when alleles of small effect control the selfing rate, selfing rates do not vary as much between inbred vs. outcrossed individuals; this results in the broader stability of mixed mating. We also find that, provided selfing is not caused by recessive alleles, low levels of outcrossing can be maintained when the average relative fitness of selfed progeny is above 0.5 . This is postulated to be due to a long-term advantage for occasional outcrossing in terms of the restoration of low inbreeding coefficients. The assumption of a monotonic decline in fitness with consecutive generations of selfing is also discussed.
\end{abstract}

Keywords: genetic transmission, inbreeding, mixed mating, self-fertilization.

\section{Introduction}

The evolutionary trajectory of a phenotypic trait can be influenced by many components of its genetic control. These genetic components include the heritability, the number of genes controlling the trait and their average effect, dominance, linkage, pleiotropy, and epistatic interactions among these genes. However, given sufficient mutational variation, the heritability, the gene effects and the degree of dominance are not expected to affect the evolutionary equilibrium of most phenotypic traits. An exception may include traits that influence the process of genetic transmission. Changes in the rules of inheritance also change the rules of the game, and hence may change the evolutionarily favoured strategy.

The theory for the evolution of inbred plant mating systems was first worked out by Fisher (1941). Since selfing individuals pass on two copies of their genes to their selfed offspring, genotypes which increase selfing are favoured if selfed offspring are greater then half as fit as outcrossed offspring; otherwise outcrossing is

*Correspondence. Present address: Department of EPO Biology, University of Colorado, Boulder, Colorado, 80309, U.S.A. advantageous (Fisher, 1941; Lloyd, 1979). This results in the prediction (Nagylaki, 1976; Wells, 1979) that only complete selfing or complete outcrossing are stable mating strategies. If genetic load is 'purged', this result is reinforced since selfed offspring have high relative fitness in selfing populations and low fitness in outcrossed populations (Lande \& Schemske, 1985; Charlesworth \& Charlesworth, 1987). However, the documentation of many partially selfing species (reviewed in Barrett \& Eckert, 1990) has motivated continued study of the factors which control the evolution of plant mating systems.

Maynard Smith (1977) suggested a possible explanation for the existence of mixed mating systems (see also Campbell, 1986; Damgaard et al., 1992). If the fitness of selfed plants relative to outcrossed plants varies according to the number of consecutive generations of selfing, selfing may be advantageous for outcrossed plants, but disadvantageous for inbred plants, resulting in stable mixed mating. For this to occur, the relative fitness of plants selfed one generation must be greater than 0.5 , while that of plants selfed many consecutive generations must be less than 0.5 . With inbreeding depression caused by overdominance at several loci (Campbell, 1986; Barrett \& Charlesworth, 1991), or 
by recessive deleterious alleles with little purging of inbreeding depression in selfing lines (Latta, 1992), one expects this relative fitness of selfed plants to decrease as a function of the number of selfed generations, at least for several generations.

In addition to these fitness changes, we note that the selfing rate, if heritable, will differ between individuals selfed for different numbers of generations. In general, the selfing rate of plants selfed for $i$ generations increases with $i$. Furthermore, if genes for selfing are recessive, the expression of these genes will depend on the number of generations of selfing. This covariation between selfing rate and inbreeding generates genetic associations among loci (Uyenoyama \& Waller, 1991a,b,c; Holsinger, 1991), and may explain Holsinger's (1988) result that the fate of an allele depends on the magnitude of its effects on selfing rate.

In this paper, we show how the equilibrium selfing rate is influenced by the genetic control of selfing. The genetic control of selfing is only one factor affecting mating strategy, and we stress that we are only examining one aspect of the problem. Maynard Smith's approach of tracking changes across consecutive generations of selfing, seems a useful approach for studying this problem. In order to highlight the difference between types of genetic control of selfing, we assume a fixed relationship between fitness and the number of consecutive generations of selfing, and neglect that this relationship itself evolves. However, this approach seems a reasonable approximation of the associations which develop between loci controlling the mating system and those affecting fitness. We examine Maynard Smith's (1977) model using iterative deterministic recursions, where selfing is subject to various modes of genetic control. We follow Maynard Smith in considering any equilibrium selfing rate exclusive of zero and one to be a mixed mating system. The following modes of genetic control are examined and compared: (i) single major additive gene; (ii) several minor additive genes; (iii) quantitative inheritance; and (iv) a single gene with dominance.

We find that selfed plants have a higher selfing rate than outcrossed plants if selfing has high genetic variance. Under these circumstances, lineages become locked into repeating the same mating type so that the conditions for stable mixed-mating are broadest when selfing has low genetic variance. We also find that dominant genes for selfing give a broader range of conditions favouring mixed-mating, compared with additive or recessive genes. Our models support Holsinger's (1988) view that a population average of the relative fitness of selfed individuals is not an adequate predictor of mating system evolution.

Theoretical studies of the genetic characteristics of inbreeding depression suggest that Maynard Smith's
(1977) fitness model, which assumes a monotonic decline of fitness with continued inbreeding, is possible only under biologically implausible circumstances (Charlesworth \& Charlesworth, 1990; Barrett \& Charlesworth, 1991). However, several empirical studies suggest that some organisms do in fact experience a steady decrease in fitness over consecutive generations of inbreeding (e.g. Hollingsworth \& Maynard Smith, 1955; Hallauer \& Sears, 1973; Schuster \& Michael, 1976; Monti \& Frusciante, 1984) for at least the first few generations of inbreeding. We therefore discuss genetic models which may give a monotonic decline of fitness with consecutive generations of selfing, and the implications of non-monotonic decline in fitness on the predictions of this model.

\section{The model}

The basic unit of observation is the fraction of plants selfed for $0,1, \ldots g$ generations, denoted by the vector $P_{i},(i=1, g)$. In our calculations, we use $g=10$. Plants in these classes have inbreeding coefficients $\left(F_{i}\right)$ of $0,1 / 2$, $3 / 4, \ldots\left[1-(1 / 2)^{g}\right]$, and the relative fitness of these plants, termed $w_{i}$, is assumed to be a function of their inbreeding coefficient (outcrossed plants have fitness $w_{0}=1$ regardless of the number of generations the parent had been selfed prior to outcrossing). Let $s_{i}\left(=1-t_{i}\right)$ be the selfing rate of plants selfed $i$ generations. These $s_{i}$ differ with $i$ if selfing is heritable. For individuals selfed $i$ generations in a population of hermaphroditic plants, a proportion $s_{i}$ will self-fertilize to produce individuals selfed $i+1$ generations. The frequency of outcrossers $\left(P_{0}\right)$ is the sum of the frequency of outcrossing plants over all generation classes $\left(t_{i}\right)$. We impose selection by multiplying the progeny frequencies, $P_{i}$, by the fitness, $w_{i}$, and normalizing by the mean fitness. Mathematically, these recursions are as follows where primes denote proportions in the following generation:

$P_{i+1}^{\prime}=P_{i} s_{i} w_{i+1} / \bar{W}$

and

$$
P_{0}^{\prime}=\Sigma P_{i} t_{i} / \bar{W}
$$

where $\bar{W}=\Sigma P_{i}\left(s_{i} w_{i}+t_{i}\right)($ the mean fitness $)$ and $w_{0}=1$.

We assume two types of relationship of $w_{i}$ with $F_{i}$ ('fitness functions'). The first is

$$
\begin{aligned}
w_{i} & =\left(1-F_{i}\right) \delta_{\infty}+\left(1-\delta_{\infty}\right) \\
& =1-\delta_{\infty} F_{i},
\end{aligned}
$$

where $\delta_{\infty}$ is the inbreeding depression shown by a plant selfed for very many consecutive generations $(F=1)$. The function specifies a linear decrease of fitness with increasing homozygosity, $F$, and depicts additive fitness interactions between loci carrying 
recessive mutations with no purging within inbred lines. The second fitness function is

$$
\begin{aligned}
w_{i} & =\left(1-F_{i}\right)^{2} \delta_{\infty}+\left(1-\delta_{\infty}\right) \\
& =1-\delta_{\infty} F_{i}\left(2-F_{i}\right) .
\end{aligned}
$$

Equation 2(b) declines monotonically and gives the same fitness at $F=1$ as eqn $2(\mathrm{a})$, but it is a concave function, wherein the fitness loss each generation becomes less severe with higher $F$. Concave fitness functions arise from partial purging and/or multiplicative fitness interactions among loci (Latta, 1992). While the fitness effects of inbreeding have been shown to depend upon the selfing genotype (Uyenoyama \& Waller, 1990a), we are here aproximating this by assuming that these differences derive from the distribution of alleles at selfing rate loci among individuals selfed for different numbers of generations. Some empirically derived fitness functions are reviewed by Damgaard et al. (1992).

In the following, we will regard $\delta_{\infty}$ as the parameter determining the rate of fitness loss over consecutive generations of selfing. This parameter can be regarded as either (i) one minus the relative fitness of a fully homozygous individual, which with $w_{0}=1$, is the inbreeding depression experienced by a highly selfed individual, or (ii) in the case of a linear fitness function, as its downward slope.

Genetic control of selfing is incorporated by calculating genótype frequencies at loci controlling selfing in plants selfed for $i$ generations ( $i=0,1, \ldots g$ generations). One can imagine each $P_{i}$ above being subdivided into a vector of genotypic frequencies (such that a matrix of genotypic frequencies is created $c f$. Table 1$)$. For con-

Table 1 Equilibrium distribution of selfing alleles among individuals selfed for $i$ consecutive generations in populations practicing mixed mating, with $\delta_{\infty}=0.55$. (a) Balanced polymorphism at one locus with two alleles of major effect. (b) Distribution of alleles of very small effect. $F$ and $W$ are the inbreeding coefficient and fitness of each generation respectively. $P_{i}$ is the proportion of individuals selfed $i$ generations, $A A, A a$ and $a a$ are the frequencies of homozygotes for outcrossing, heterozygotes, and homozygotes for selfing alleles, respectively (such that $\left.A A+A a+a a=P_{i}\right)$. The overall frequency of the selfing allele in each class is $q$, and

\begin{tabular}{|c|c|c|c|c|c|c|c|c|}
\hline$i$ & $F$ & $W$ & $P$ & $A A$ & $A a$ & $a a$ & $q$ & $s$ \\
\hline \multicolumn{9}{|l|}{ (a) } \\
\hline 0 & 0.000 & 1.000 & 0.284 & 0.057 & 0.168 & 0.057 & 0.500 & 0.499 \\
\hline 1 & 0.500 & 0.725 & 0.204 & 0.030 & 0.060 & 0.113 & 0.704 & 0.703 \\
\hline 2 & 0.750 & 0.587 & 0.149 & 0.007 & 0.015 & 0.126 & 0.895 & 0.894 \\
\hline 3 & 0.875 & 0.518 & 0.113 & 0.001 & 0.003 & 0.108 & 0.971 & 0.970 \\
\hline 4 & 0.937 & 0.484 & 0.082 & 0.000 & 0.000 & 0.081 & 0.993 & 0.992 \\
\hline 5 & 0.968 & 0.467 & 0.056 & 0.000 & 0.000 & 0.056 & 0.999 & 0.998 \\
\hline 6 & 0.984 & 0.458 & 0.038 & 0.000 & 0.000 & 0.038 & 1.000 & 0.999 \\
\hline 7 & 0.992 & 0.454 & 0.025 & 0.000 & 0.000 & 0.025 & 1.000 & 0.999 \\
\hline 8 & 0.996 & 0.452 & 0.013 & 0.000 & 0.000 & 0.013 & 1.000 & 0.999 \\
\hline 9 & 0.998 & 0.451 & 0.007 & 0.000 & 0.000 & 0.007 & 1.000 & 0.999 \\
\hline 10 & 0.999 & 0.450 & 0.006 & 0.000 & 0.000 & 0.006 & 1.000 & 1.000 \\
\hline \multicolumn{9}{|c|}{ Mean selfing rate $=0.715$. Average $\delta=0.424$} \\
\hline \multicolumn{9}{|l|}{ (b) } \\
\hline 0 & 0.000 & 1.000 & 0.354 & 0.067 & 0.174 & 0.113 & 0.434 & 0.645 \\
\hline 1 & 0.500 & 0.725 & 0.300 & 0.094 & 0.074 & 0.131 & 0.439 & 0.645 \\
\hline 2 & 0.750 & 0.587 & 0.184 & 0.070 & 0.022 & 0.091 & 0.444 & 0.645 \\
\hline 3 & 0.875 & 0.518 & 0.092 & 0.038 & 0.005 & 0.047 & 0.451 & 0.645 \\
\hline 4 & 0.937 & 0.484 & 0.040 & 0.017 & 0.003 & 0.008 & 0.458 & 0.645 \\
\hline 5 & 0.968 & 0.467 & 0.016 & 0.007 & 0.000 & 0.008 & 0.465 & 0.645 \\
\hline 6 & 0.984 & 0.458 & 0.006 & 0.003 & 0.000 & 0.003 & 0.473 & 0.645 \\
\hline 7 & 0.992 & 0.454 & 0.002 & 0.001 & 0.000 & 0.001 & 0.480 & 0.646 \\
\hline 8 & 0.996 & 0.452 & 0.001 & 0.000 & 0.000 & 0.000 & 0.488 & 0.646 \\
\hline 9 & 0.998 & 0.451 & 0.001 & 0.000 & 0.000 & 0.000 & 0.511 & 0.646 \\
\hline 10 & 0.999 & 0.450 & 0.000 & 0.000 & 0.000 & 0.000 & 0.523 & 0.646 \\
\hline \multicolumn{9}{|c|}{ Mean selfing rate $=0.645$. Average $\delta=0.372$} \\
\hline
\end{tabular}
$s$ is the selfing rate among plants selfed $i$ generations 
secutive generations of selfing, these genotypic frequencies are calculated from the rules of Mendelian segregation. For example, at a single locus, the frequency of heterozygotes selfed $i$ generations equals half the frequency of heterozygotes selfed $i-1$ generations times the selfing rate of heterozygotes. To calculate genotype frequencies forming the newly outcrossed generation we first find gene frequencies separately among the outcrossed pollen and ovule pools. Ovule gene frequencies are weighted by $t_{i} P_{i}$ for generation $i$. Genotype frequencies among outcrossed plants are then calculated as the product of the pollen and ovule gene frequencies. Ordinarily, one assumes that selfing plants contribute fully to the outcrossing pollen pool, but we also considered the case of partial contribution (pollen discounting), as discussed below.

\section{Results of genetic models of selfing}

\section{Single major gene}

We first modelled selfing rate as controlled by a single gene with two alleles, and no dominance or environmental effects. The alternative homozygotes were completely selfing and completely outcrossing, and heterozygotes had selfing rate 0.5 . The vector of gene frequencies was initialized in Hardy-Weinberg proportions with both allele frequencies equal to 0.5. Equilibria were found to be independent of the starting allele frequencies. The equilibrium frequency distribution of genotype was obtained by iterating (1) until genotypic frequencies, and hence the selfing rate, stabilized. The equilibrium selfing rate was evaluated over a range of possible values for $\delta_{\infty}$.

With one locus controlling selfing and a linear fitness function, values of $\delta_{\infty}$ between 0.4 and 0.85 result in equilibrium mixed-mating (Fig. 1a). However, with a curved fitness function, this range is substantially reduced to between 0.4 and 0.65 (Fig. $1 \mathrm{~b}$ ).

At equilibrium, the distribution of individuals selfed for $i$ generations is skewed toward low $i$, even for quite high selfing rates (Table 1a). Few indivdiuals have resulted from more than a few consecutive generations of selfing. With selfing controlled by a single polymorphic locus, the frequency of selfing alleles is markedly different between outcrossed and fully selfed individuals. Thus, the selfing rate rises sharply towards one as the number of generations of selfing increases. Also, the level of inbreeding depression averaged over all individuals (as normally measured empirically) is less than 0.5 which would suggest that the selfing alleles should increase in frequency. Nonetheless, the situation depicted in Table $1(a)$ is in equilibrium.

\section{Several minor genes}

We modelled several minor additive genes controlling the selfing rate by assuming that genotypes showed a binomial distribution of selfing rates, with the extremes of the distribution at complete selfing and complete outcrossing. Each selfing allele increased the selfing rate by $0.5 / n$, where $n$ is the number of loci. With more loci controlling the selfing rate, the range of $\delta_{\infty}$ allowing mixed mating is increased: with four loci, values of $\delta_{\infty}$ between 0.3 and 1.0 give mixed-mating (Fig. 1a and b).

\section{Alleles of small effect}

We modelled small allelic effects by examining one locus with two codominant alleles specifying very similar selfing rates, $s$ and $s+0.01$. If the selfing allele was eliminated then the equilibrium selfing rate was lower than $s$. Likewise, if the outcrossing allele was eliminated, the equilibrium selfing rate was higher than $s+0.01$. We searched for pairs of alleles which could both be maintained in the population for a given $\delta_{\infty}$. This gave the equilibrium selfing rate which would be expected in a population in which all plants produced an equal proportion of selfed offspring, with no noticeable polymorphisms for selfing rate.

Figure 1(a) and (b) show the equilibrium for the small effects' model. Evolution of selfing by mutations of small effect gives the greatest range of $\delta_{\infty}$ for which mixed mating is stable. Since the range of $\delta_{\infty}$ for which mixed mating is stable increases with the number of loci, this analysis seems the best approximation for a large number of loci of small effect. The range of stable mixed-mating systems spans $0.25<\delta_{\infty}<1.0$ for linear and $0.35<\delta_{\infty}<0.7$ for curved fitness functions (Fig. $1 \mathrm{a}$ and $\mathrm{b})$.

The distribution of gene frequencies across consecutive generations of selfing under the 'small effects' model is given in Table 1 (b). The difference in allele frequencies at the selfing locus, and hence in selfing rate, is much less between outcrossed and highly selfed individuals than it is under single locus inheritance. The distribution of individuals selfed for different numbers of generations $\left(P_{i}\right)$ is more markedly skewed toward low $i$ than with genes of major effect. As a result, the equilibrium selfing rate is different for the same function.

\section{A quantitative genetic model}

A different approach is to model selfing as a trait showing continuous inheritance. Assume a plant derived from selfing $i$ generations has selfing phenotype $\mathbf{P}_{i}$, and 


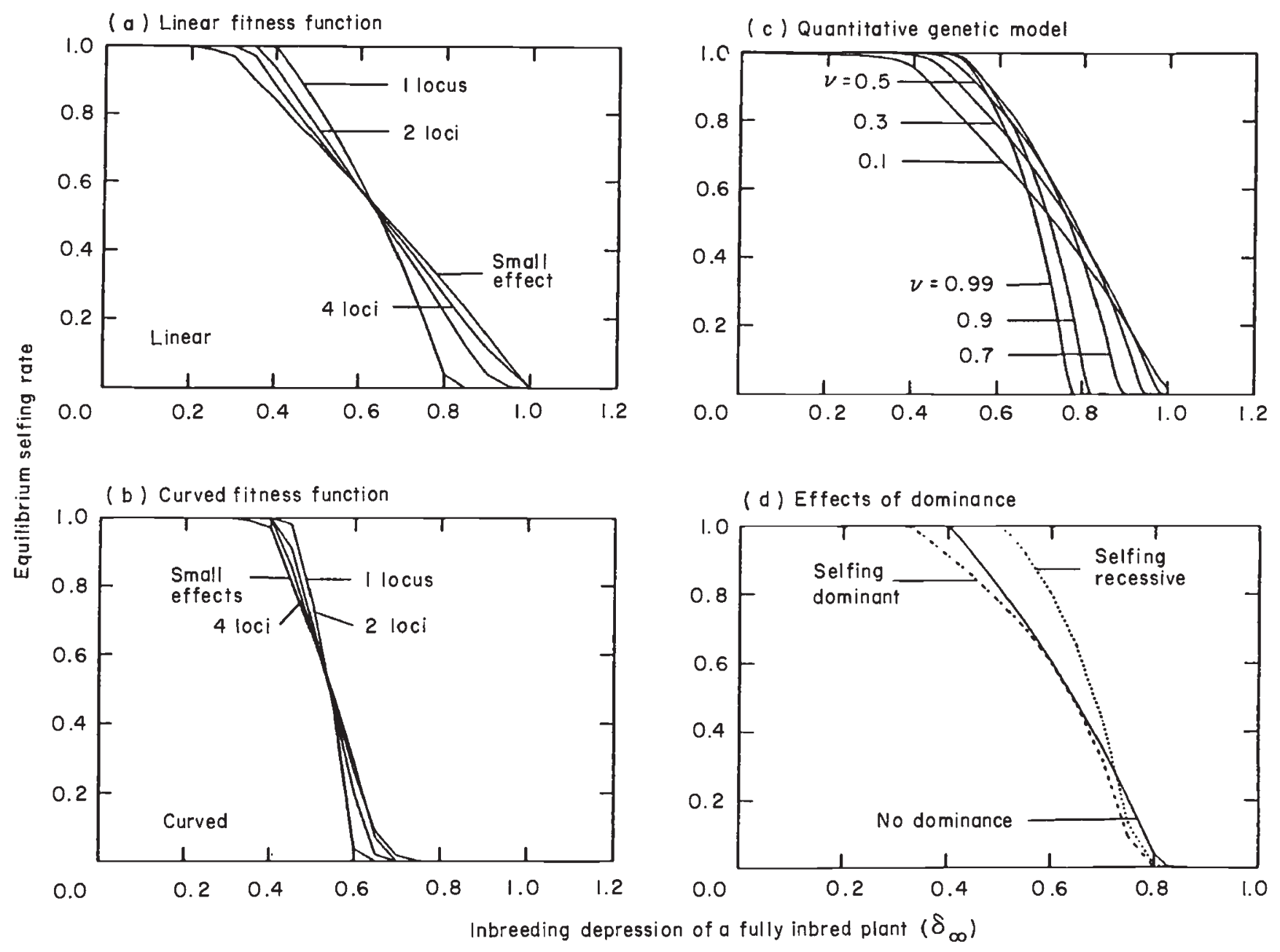

Fig. 1 Equilibrium selfing rates under different modes of inheritance: (a) single locus inheritance with a linear fitness function; (b) single locus inheritance with a curved fitness function; (c) continuous inheritance with a linear fitness function; (d) single locus with additive, dominant, and recessive alleles for selfing, and a linear fitness function.

that this phenotype is the sum of independent genetic and environmental contributions: $\mathbf{P}_{i}=\mathbf{G}_{i}+\mathbf{E}_{i}$. These quantities are random variables, each of which follow a certain distribution; the environmental effect is assumed to have zero mean, so that the mean phenotype equals the mean genotype, denoted as $\mu_{i}$. Let $\sigma_{i}^{2}$ be the genetic variance for selfing in generation $i$. At equilibrium, the selfing rate in generation $i+1$ is the expected selfing genotype among selfing phenotypes in generation $i$, divided by the expected amount of selfing in generation $i$,

$$
\begin{aligned}
\mu_{i+1} & =\mathrm{E}\left[\mathbf{G}_{i} \mathbf{P}_{i}\right] / \mathrm{E}\left[\mathbf{P}_{i}\right] \\
& =\left(\mu_{i}^{2}+\sigma_{i}^{2}\right) / \mu_{i} \\
& =\mu_{i}+\left(1-\mu_{i}\right) \nu_{i},
\end{aligned}
$$

where $E[.$.$] denotes expected value and$

$$
v_{i}=\sigma_{i}^{2} /\left[\mu_{i}\left(1-\mu_{i}\right)\right]
$$

is the normalized genetic variance of selfing among individuals selfed $i$ generations. The relation $\mathrm{E}\left[\mathbf{G}_{i} \mathbf{P}_{i}\right]=\mu_{i}^{2}+\sigma_{i}^{2}$ holds because there is no covariance between genetic and environmental effects, so $\mathrm{E}\left[\mathbf{G}_{i} \mathbf{P}_{i}\right]=\mathrm{E}\left[\mathbf{G}_{i}^{2}\right]$.

This normalization of genetic variance by $\mu(1-\mu)$ removes much of the dependence of genetic variance upon the selfing rate. For example, if selfing rate is controlled by $n$ diploid loci, each with additive alleles and all with equal effect, the un-normalized genetic variance would be proportional to the binomial variance $\mu(1-\mu) / n$, while the normalized genetic variance would be proportional to $1 / n$.

To find $\mu_{0}$, we need to compute the genetically programmed selfing rate in gametes by outcrossing plants. Among outcrossing females of class $i$, this selfing rate is the expected frequency of selfing genes among outcrossing individuals, divided by the expected 


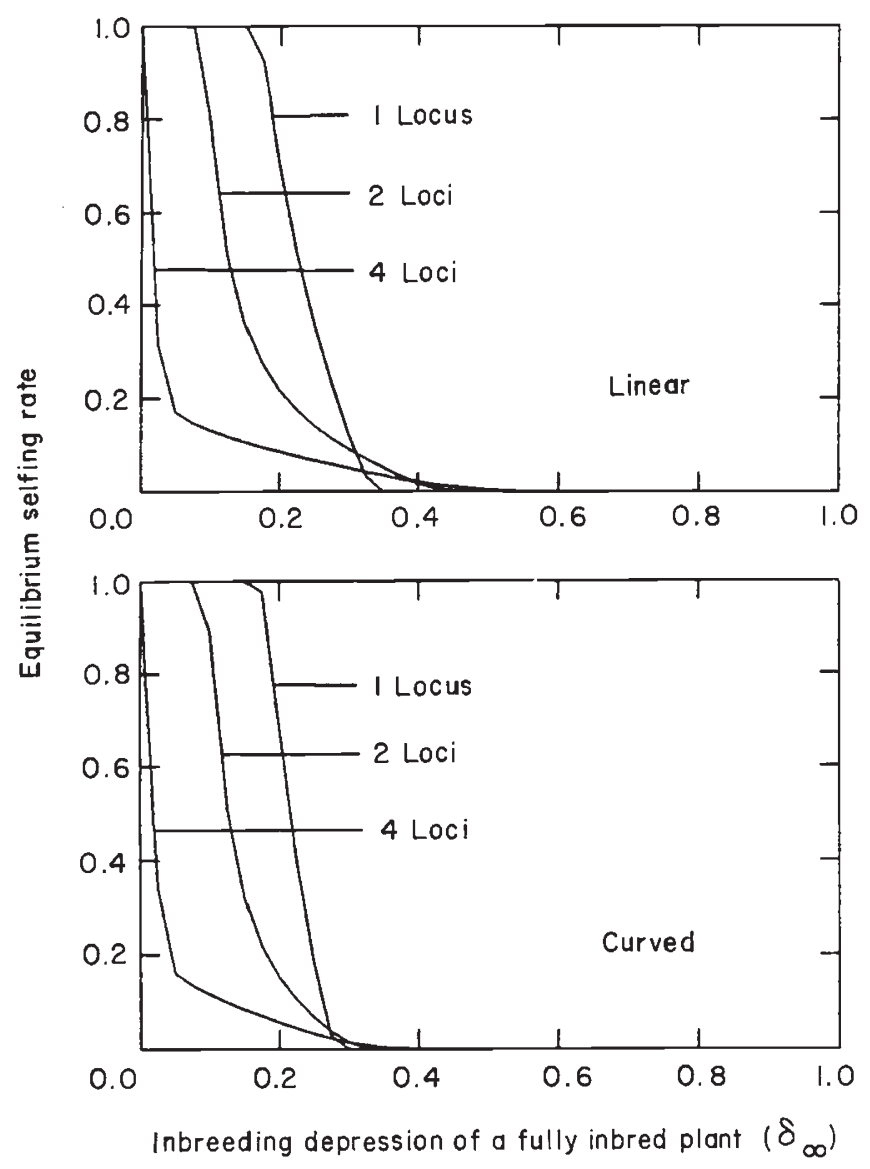

Fig. 2 Equilibrium selfing rates with 50 per cent pollen discounting: (a) linear fitness function; (b) curved fitness function.

outcrossing rate,

$$
\begin{aligned}
\mu_{0}(\text { female gametes }) & =\mathrm{E}\left[\mathbf{G}_{i}\left(1-\mathbf{P}_{i}\right)\right] / \mathrm{E}\left[1-\mathbf{P}_{i}\right] \\
& =\mu_{i}\left(1-v_{i}\right) .
\end{aligned}
$$

Among outcrossing males of class $i$, this selfing rate is a mixture of the above and $\mu_{i}$,

$\mu_{0}($ male gametes $)=(1-d) \mu_{i}+d \mu_{i}\left(1-v_{i}\right)$,

where $d$ is the pollen discounting, i.e. the proportion by which outcrossing pollen is reduced when a plant selfs. The mean selfing rate among outcrossed plants is the arithmetic average among males and females, summed over all classes $i$, or

$\mu_{0}=\Sigma P_{i} \mu_{i}\left(1-[(1+d) / 2] v_{i}\right)$,

where the $P_{i}$ are defined as in eqn (1). These $P_{i}$ are then iterated as described before to obtain equilibrium selfing rates in the population.

When selfing was modelled in this way under a linear fitness function, the mixed-mating equilibria, shown in Fig. 1(c), showed patterns similar to Fig. 1(a).
Under a curved fitness function, a similar correspondence to Fig. 1(b) was also observed (not shown). When the normalized genetic variance is low $(0.1)$, the patterns of equilibria are close to those found by the ESS model $\left(\delta_{\infty}\right.$ between 0.25 and 1.0 gave mixedmating), while when the normalized genetic variance is high $(0.99)$, equilibria are close to the one-locus model prediction $\left(\delta_{\infty}\right.$ between 0.40 and 0.85 resulted in mixed mating). However, the correspondence is not exact, as shown by an apparent 'curvature' of the equilibria surface in Fig. 1(c) not evident in Fig. 1(a). When the effect of inbreeding was also included by specifying the normalized genetic variance for selfing in generation $i$ as $v_{i} /\left(2-F_{i}\right)$, where $F_{i}=1-(1 / 2)^{i}$ is the inbreeding coefficient of individuals selfed $i$ generations, the correspondence to the previous models became much closer.

Interestingly, the magnitude of environmental variation does enter into these quantitative genetic recursions; this is probably due to the linear (as opposed to threshold) selection of selfed individuals for succeeding generations. Equilibria depend on the heritability only through the magnitude of the genetic variance.

\section{Dominance}

We modified the model of a single major locus to allow for dominance, with selfing being either completely dominant or completely recessive, and assumed the linear fitness function. When selfing is recessive, the range of $\delta_{\infty}$ giving mixed mating is much reduced relative to the model with no dominance, and complete selfing occurs for all $\delta_{\infty}<0.5$ (Fig. 1d). In contrast, when selfing is dominant, the range of $\delta_{\infty}$ allowing mixed mating is expanded relative to the no dominance case. Also, mixed mating was stable for values of $\delta_{\infty}$ well below 0.5 , and complete selfing was less likely when dominant than when recessive.

\section{Pollen discounting}

Genotypes that self may suffer from a reduction of pollen available for outcrossing ('pollen discounting' $c f$. Holsinger et al., 1984). To allow for this possibility, we incorporated pollen discounting by reducing the pollen contribution of genotypes by $d s_{i}$, where $s_{i}$ is the selfing rate of plants selfed $i$ generations. The parameter $d$ is the proportionate reduction in pollen available for outcrossing in a plant which selfs completely. The parameter $d$ ranges from 0 (no discounting) to 1 (complete discounting). We assumed equal $d$ among all genotypes.

Under all modes of inheritance, pollen discounting reduced the range of $\delta_{\infty}$ allowing mixed-mating (Fig. 
$2 a$ and $b)$. The effect is roughly linear with increasing discounting. With high pollen discounting, selfing is almost never stable and the range of stable mixedmating systems is small. Also, as noted by previous workers (e.g. Lloyd, 1979), pollen discounting raised the equilibrium outcrossing rate (i.e. curves shift to the left with increasing $d$ ).

\section{Discussion}

Maynard Smith (1977), in establishing conditions for equilibrium partial selfing, considered the case of a recessive allele for full selfing, and solved for conditions which would permit both selfing and outcrossing alleles to be maintained in the population. He assumed that the results of his model held for any mechanism of genetic control of selfing. When explicit genetic models are compared, we find that the genetic control of selffertilization can have a major effect on the equilibrium selfing rate. Equilibrium selfing rates can differ by as much as 0.3 between different genetic models (compare single loci of large and small effect for $\delta_{\infty}=0.45$ in Fig. 1a). In addition, the range of conditions allowing mixed-mating are greatly dependent on the mode of genetic control. We found mixed mating to be less likely (i) when selfing has high genetic variance, such as when controlled by a single major gene of large effect (Fig. 1a-c); (ii) when selfing is recessive (Fig. 1d); or (iii) when pollen discounting is allowed (Fig. 2).

The classical condition for an outcrossing population to resist invasion of a selfing allele is that the fitness of plants selfed for one generation must be less than one half that of outcrossed plants. In these calculations, this corresponds to $\delta_{\infty}>1.0$ for the linear fitness function and $\delta_{\infty}>0.67$ for the curved function. This prediction was confirmed by us, as in no case did we observe mixed-mating systems for values of $\delta_{\infty}$ greater than these. However, our results differ from Maynard Smith's conditions $\left(\delta_{\infty}<0.5\right)$ for the complete elimination of outcrossing from the population. Maynard Smith's prediction for a single recessive selfing gene was confirmed but with polygenic control of selfing, outcrossing rates of at least 10 per cent were observed for values of $\delta_{\infty}$ as low as 0.3 (Fig. 1a). When $\delta_{\infty}<0.5$, all selfed plants are at least half as fit as outcrossed plants, and we expect selfing to be favoured even in fully inbred plants. That some genetic models permit outcrossing to be maintained under this condition underscores the importance of the genetic control of selfing to the equilibrium mating system.

It may at first seem unexpected that the genetic control of selfing influences the equilibrium selfing rate. However, the reason for this becomes evident if we consider the distribution of selfing genotypes among plants selfed $0,1,2 \ldots g$ generations (Table 1 ). If selfing rate is heritable, the selfing rate among individuals selfed $i$ generations increases with increasing $i$, and this difference is more pronounced when the selfing rate has high genetic variance such as with a polymorphism of selfing and outcrossing morphs. As this difference becomes more pronounced, outcrossing alleles occur primarily in high fitness outcrossed plants and selfing alleles primarily in low fitness inbred plants. Thus, the selection differential between selfing and outcrossing alleles becomes stronger, and mixed mating systems become less likely.

Another way to understand how the genetic control of selfing influences its equilibrium value is to consider the evolution of selfing in terms of competition between genotypes of different inbreeding coefficients (and not in the traditional terms of competition between selfed and outcrossed genotypes). In this conception, if the fitness of selfed progeny declines with $F$, then selfed progeny of plants selfed for few generations are favoured over selfed progeny of plants selfed for many generations. Occasional outcrossing is disadvantageous in the first generation because of the cost of meiosis, but, over the longer term this cost is offset by the advantage of lower breeding coefficients in subsequently selfed lines of grand progeny.

Under this interpretation, the genetic control of selfing assumes importance in the following way. With few genes of major effect, outcrossed plants are less likely to subsequently produce selfed progeny; instead, outcrossed genotypes are 'locked' into outcrossing, and suffer repeated costs of meiosis, resulting in narrowed conditions for mixed mating. Likewise, selfing genotypes are locked into low fitness inbred plants. By contrast, if genetic variance for selfing is low (due to many genes of small effect), selfing genotypes can occasionally outcross without risk of becoming locked into outcrossing. The long-term benefit of outcrossing in terms of lowered inbreeding coefficients in selfing lines is realized, and mixed mating becomes a stable strategy of reproduction.

The maintenance of some trace amount of outcrossing in populations with very low inbreeding depression $\left(\delta_{\infty}<0.5\right)$ results from these long-term benefits of outcrossing. But it is only possible if the genetic control of selfing prevents outcrossing genotypes from being locked into outcrossing. If selfing is recessive, the outcrossed progeny, which will usually be heterozygous at the selfing locus, will tend to outcross again. Thus the advantage of occasional outcrossing does not occur, and mixed mating is not observed for $\delta_{\infty}<0.5$. If selfing alleles are dominant, however, then all outcrossed progeny (the progeny of rare recessive parents) will be heterozygous at selfing loci. They will produce selfed 
offspring, and the advantage of occasional outcrossing is realized. Consequently, some outcrossing is maintained even at very low values of $\delta_{\infty}$ when selfing is dominant.

\section{Implications for mating system evolution}

Our results show that mixed mating systems are more likely to occur as a monomorphic optimal strategy under polygenic control, than as a balanced, singlelocus polymorphism. A population in which all individuals produce an equal proportion of selfed offspring with little variation (or in which each plant selfs with equal probability) is more likely to be stable than a population in which a proportion of the individuals self-fertilize and the rest outcross. The range of $\delta_{\infty}$ permitting mixed mating is widest when the genetic basis of the mating system is polygenic. This result suggests that such genetic control may be the most common system among natural populations practising mixed mating. By the same reasoning, selfing alleles within mixed-mating populations are more likely to be of additive or dominant effect, as opposed to recessive, since such effects also give a wide range of $\delta_{\infty}$ allowing mixed-mating (Fig. 1d). Conversely, selfing taxa could likely be derived from the fixation of recessive genes, as opposed to genes with additive or dominant effects, as recessive inheritance shifts equilibria towards higher selfing (Fig. 1d). Our observations regarding the narrowing of conditions favouring mixed mating under pollen discounting also suggest that substantial pollen discounting is not to be expected in habitually mixedmating species. Likewise, our findings suggest that mixed mating systems should arise more frequently when fitness shows a linear decrease with increasing $F$.

Few workers have attempted to document the genetic basis of selfing in partially selfing species, or have attempted to document the genetic basis for differences between con-generic selfing and outcrossing species. The few studies which have been performed tend to be with genera with conspicuous intrapopulation polymorphisms, such as Senecio (Marshall \& Abbott, 1982, 1984) and Eichhornia (Barrett et al., 1989). These systems usually show major gene control (e.g. Impomoea purpurea, Ennos \& Clegg, 1983), and provide tractable systems for experiments. Since we have found broader conditions favouring mixed mating under polygenic (as opposed to single gene) models, polygenic control of the selfing rate seems more likely in documented mixed mating species such as Mimulus (Ritland \& Ganders, 1987; Ritland \& Ritland, 1990) and Turnera ulmifolia (Shore \& Barrett, 1990). Genetic studies of selfing in such taxa have been rare (Barrett \& Eckert, 1990).
Holsinger (1988) noted that the average inbreeding depression in a population does not adequately predict the course of mating system evolution. The population average levels of inbreeding depression in Table $1(\delta<0.5)$ confirm this result. Though one would expect that the selfing rate will increase, the equilibria shown are stable. The reason for this is that the selfing allele occurs at higher frequency among the highly selfed, less fit individuals where they experience stronger selective disadvantage than would be predicted by the average level of inbreeding depression in the population. However, the average fitness of the selfing and outcrossing alleles summed over each generation class, is exactly equal at equilibrium.

Stebbins (1950) suggested that mating systems strike a balance between the immediate fitness of the individual, which favours selfing, and the long-term fitness of the population, which favours outcrossing. However, few models have presented explicit mechanisms of this process. The maintenance of outcrossing even at very low values of $d$ is due to a long-term benefit in terms of the number of grand progeny produced. Both Holsinger (1988) and Campbell (1986) noted that mixed mating systems could be stable for values of $\delta$ (population average in their models) substantially less than 0.5 , and in one case when $\delta$ was as low as 0.01 . Their results can be explained by the competition among individuals of different inbreeding coefficients outlined above. The maintenance of traits which promote outcrossing in predominantly selfing species (e.g. Motton \& Antonovics, 1992), and the apparent lack of completely selfing species (Waller, 1986) may reflect this advantage to occasional outcrossing. It is not necessary to resort to species selection arguments to explain these findings.

An important role in mating system evolution has recently been ascribed to the associations that develop between loci which modify the selfing rate and genotypes at loci causing inbreeding depression. Uyenoyama \& Waller (1991a,c; see also Holsinger, 1988, 1991) demonstrated that alleles which increase the selfing rate may become associated with high fitness genotypes at loci carrying deleterious recessive mutations. They also showed (Uyenoyama \& Waller, $1991 \mathrm{~b}$ ) that selfing alleles can become associated with lower fitness genotypes at loci which show overdominance. These associations derive from the distribution of selfing alleles across individuals selfed for different numbers of consecutive generations (Table 1). Uyenoyama \& Waller assumed alleles of small effect at loci controlling selfing. Since there are greater differences between outcrossed and selfed individuals when genes of large effect control the selfing rate (Table 1), 
these associations will be more pronounced for alleles with a large effect on the selfing rate.

\section{The fitness functions}

Our predictions rest upon the model suggested by Maynard Smith (1977), which postulates that the fitness of selfed progeny decreases with increased inbreeding coefficients of parents. In order for mixed mating systems to be stable the fitness of plants selfed for many generations must be less that that of plants selfed one generation. Given this consideration, our result that the range of $\delta_{\infty}$ allowing mixed mating systems should be narrowed with a curved fitness function is easily understood. If fitness functions are curved, more than half of the total fitness loss occurs in the first generation of selfing, and the subsequent loss of fitness with continued selfing is reduced. In other words, the curved fitness function is closer to the usual assumption of constant $\delta$ for all selfed offspring, which does not allow mixed mating.

The relationship between fitness and the number of consecutive generations of selfing will be governed by two factors: (i) the decrease in heterozygosity with successive generations of selfing; and (ii) the removal of genetic load through purging. If purging is such that the fitness of individuals selfed for many generations is greater than 0.5 , the predictions of our model will not hold for a deterministic calculation such as ours. However, if purging is slow enough that fitness does not recover within inbred lines $(<10$ generations) then the model will hold.

Barrett \& Charlesworth (1991) calculated the expected fitness function under a number of models of inbreeding depression. Overdominance at a few loci can lead to declining fitness functions. Moreover, mixed mating systems can be stable with overdominance fitness models (Charlesworth \& Charlesworth, 1990). Conversely if inbreeding depression is due to recessive lethal mutations at a few loci, then purging is rapid and the fitness of highly inbred individuals often exceeds that of outcrossed plants. Mixed mating systems are not stable for these conditions (Charlesworth et al., 1990). With less intense selection against the mutations, however, the rate of purging slows, but fitness will nonetheless recover. Extending Barrett \& Charlesworth's model to many mutations with very small fitness effects, purging will not appreciably affect fitness for many consecutive generations of selfing (Latta, 1992). Moreover, with very many recessive mutations, the assumption that mutations are unlinked will not be met. When mutations are linked in repulsion phase, linkage blocks are effectively overdominant, and purging may be greatly slowed (Jones, 1917, 1957).
Theories about inbreeding depression are of limited use without empirical studies of the genetic architecture of load (e.g. Hedrick \& Muona, 1990). Several empirical data sets suggest that fitness does indeed continue to decline with consecutive generations of selfing, at least in some organisms. Hollingsworth \& Maynard Smith (1955) followed 16 generations of sib mating in Drosophila melanogaster, and demonstrated that purging of genetic load did not begin until 14 or 15 generations. Wilton et al. (1989) have also demonstrated that purging in Drosophila is weak enough to take many generations. Damgaard data from several plant species which show monotonically declining fitness functions. Moreover, many of these species practise mixed mating. and Damgaard et al. (1992) have shown that their selfing rates are accurately predicted by Maynard Smith's model.

\section{Acknowledgements}

We would like to thank Josh Kohn, Dan Schoen, Chris Eckert, and Brian Husband for helpful comments on earlier drafts of this manuscript, and to two reviewers for useful comments. This work was supported by a graduate scholarship to Robert Latta from the Natural Sciences and Engineering Research Council of Canada and an NSERC operating grant to Kermit Ritland.

\section{References}

BARRETT, s. C. H. AND CHARLESWORTH, D. 1991. Effects of a change in the level of inbreeding on the genetic load. Nature, 352, 522-524.

BARRETT, S. C. H. AND ECKERT, C. G. 1990. Variation and evolution of plant mating systems. In: Kawano, S. (eds.) Biological Approaches and Evolutionary Trends in Plants. Academic Press, London, pp. 229-254.

BARRETT, s. C. H., MORGAN, M. T. AND HUSBAND, B. C. 1989. The dissolution of a complex genetic polymorphism: the evolution of self-fertilization in tristylous Eichhornia paniculata. Evolution, 43, 1398-1416.

CAMPBELL, R. B. 1986. The interdependence of mating structure and inbreeding depression. Theor. Pop. Biol., 30, 232-244.

CHARLESWORTH, D. AND CHARLESWORTH, B. 1987. Inbreeding depression and its evolutionary consequences. Ann. Rev. Ecol. Syst., 18, 237-268.

CHARLESWORTH, D. AND CHARLESWORTH, B. 1990. Inbreeding depression with heterozygote advantage and its effect on selection for modifiers changing the outcrossing rate. Evolution, 44, 870-888.

CHARLESWORTH, D., MORGAN, M. T. AND CHARLESWORTH, B. 1990. Inbreeding depression, genetic load and the evolution of outcrossing rates in a multilocus system with no linkage. Evolution, 44, 1469-1489. 
DAmGaARD, D., COUVET, D. AND LOESCHCKe, v. 1992. Partial selfing as an optimal mating strategy. Heredity, 69, 289-295.

ENNOS, R. A. AND CLEGG, M. T. 1983. Flower colour variation in the morning glory, Ipomoea purpurea. J. Hered., 72, $247-250$.

FISHER, R. A. 1941. Average excess and average effect of a gene substitution. Ann. Eugen., 11, 53-63.

HEDRICK, P. W. AND MUONA, O. 1990. Linkage of viability genes to marker loci in selfing organisms. Heredity, 64, 67-72.

HALLAUER, A. R. AND SEARS, J. H. 1973. Changes in quantitative traits associated with inbreeding in a synthetic variety of maize. Crop Sci., 13, 327-330.

HOLLINGSWORTH, M. J. AND MAYNARD SMITH, J. 1955. The effects of inbreeding on rate of development and on fertility in Drosophila subobscura. J. Genet., 53, 295-314.

HOLSINGER, K. E. 1988. Inbreeding depression doesn't matter: the genetic basis of mating system evolution. Evolution, 42, 1235-1244.

HOLSINGER, K. E. 1991. Inbreeding depression and the evolution of plant mating systems. T.R.E.E., 6, 307-308.

HOLSINGER, K. E., FELDMAN, M. W. AND ChristianSEN, F. B. 1984. The evolution of self-fertilization in plants: a population genetic model. Am. Nat., 124, 446-453.

JONES, D. F. 1917. Dominance of linked factors as a means of accounting for heterosis. Genetics, 2, 466-479.

JONES, D. F. 1957. Gene action in heterosis. Genetics, 42, 93-103.

LANDE, R. AND SCHEMSKE, D. W. 1985. The evolution of self fertilization and inbreeding depression in plants. I. Genetic models. Evolution, 39, 24-40.

LATTA, R. G. 1992. Inbreeding Depression and Mixed Mating Systems in Mimulus. M.Sc. Thesis, University of Toronto.

LLOYD, D. G. 1979. Some reproductive factors affecting the selection of self fertilization in plants. Am. Nat., 113, 67-79.

MARSHALL, D. F. AND ABBOTT, R. J. 1982. Polymorphism for outcrossing frequency at the ray floret locus in Senecio vulgaris L. I. Evidence. Heredity, 48, 227-235.

MARSHALL, D. F. AND ABBOTT, R. J. 1984. Polymorphism for outcrossing frequency at the ray floret locus in Senecio vulgaris L. II. Confirmation. Heredity, 52, 331-336.

MAYNARD-SMITH, J. 1977. The sex habit in plants and animals. In: Christiansen, F. B. and Fenchel, T. M. (eds) Measuring Selection in Natural Populations. Springer-Verlag, Berlin, pp. 265-273.
MONTI, L. M. AND FRUSCIANTE, L. 1984. Selection methods for yield improvement in faba beans. In: Hebblethwaite, P. D. et al. (eds) Vicia faba: Agronomy, Physiology and Breeding. Dr W. Junk Publishers, The Hague.

MOTTON, A. F. AND ANTONOVICS, J. 1992. Determinants of outcrossing rate in a predominantly self-fertilizing weed, Datura stramonium (Solanaceae). Am. J. Bot., 74, 419-427.

NAGYLAKI, T. 1976. A model for the evolution of self-fertilization and vegetative reproduction. J. Theoret. Biol., 58, 55-58.

RITLAND, K. M. AND GANDERS, F. R. 1987. Covariation of selfing rates with parental gene fixation indices within populations of Mimulus guttatus. Evolution, 41, 760-771.

RITLAND, C. AND RITLAND, K. M. 1990. Variation of sex allocation among eight taxa of the Mimulus guttatus species complex (Scrophulariaceae). Am. J. Bot., 76, 1731-1740.

SCHUSTER, W. AND MICHAEL, J. 1976. Untersuchungen uber Inzuchtdepression und Heterosiseffekte bei Raps (Brassica napus oleifera). Zeitschrift fur Planzenzuchtung., 77, 56-66.

SHORE, J. S. AND BARRETT, S. C. H. 1990. Quantitative genetics of floral characters in homostylous Turnera ulmifolia var. angustifolia Wild. (Turneraceae). Heredity, 64, 105-112.

Stebbins, G. L. 1950. Variation and Evolution in Plants. Columbia University Press, New York.

UYENOYAMA, M. K. AND WALLER, D. M. 1991a. Coevolution of self-fertilization and inbreeding depression. I. Mutationselection balance at one and two loci. Theor. Pop. Biol., 40, 14-46.

UYENOYAMA, M. K. AND WALleR, D. M. 1991b. Coevolution of self-fertilization and inbreeding depression. II. Symmetric overdominance in viability. Theor. Pop. Biol., 40, 46-77.

UYENOYAMA, M. K. AND WALKER, D. M. 1991c. Coevolution of self-fertilization and inbreeding depression. III. Homozygous lethal mutations at multiple loci. Theor. Pop. Biol., 40, 173-210.

WALLER, D. M. 1986. Is there disruptive selection for selffertilization? Am. Nat., 128, 421-426.

WELLS, H. 1979. Self-fertilization: Advantageous or deleterious. Evolution, 33, 252-255.

WILTON, A. N., JOSEPH, M. G. AND SVED, J. A. 1989. Can chromosomal heterosis in Drosophila be explained by deleterious recessive genes? Negative results from a dichromosomal population test. Genet. Res. Camb., 53, 129-140. 